\title{
Erratum to: Algebraic Order Bounded Disjointness Preserving Operators and Strongly Diagonal Operators
}

\author{
Karim Boulabiar, Gerard Buskes and Gleb Sirotkin
}

\section{Erratum to: Integr. Equ. Oper. Theory 54 (2006), 9-31 DOI 10.1007/s00020-004-1335-3}

An error in Theorem 5.3 of the original publication is corrected here which results in an added condition in Corollary 5.5 and an open problem on whether the extra condition is needed.

The error forces a deletion in a part of what was asserted with the deletion of the last few lines of its proof, and an added hypothesis to Corollary 5.5 (Corresponding changes in the introduction are left to the reader). More precisely, the proof of Theorem 5.3 was sequenced in 7 steps and the error occurred after line 4 of Step 7. A counterexample to the original theorem in question will be provided. As far as the results of the paper, the change in Theorem 5.3 only affects Corollary 5.5, which leads to an open problem. To facilitate the two corrections in the content that need to be made we introduce the following terminology.

Definition 1. Let $L$ be a vector lattice and let $F$ be a vector subspace of $L$. A linear map $T: F \rightarrow L$ is called order bounded if for every subset $A$ of $F$ that is order bounded in $L$ it follows that $T(A)$ is order bounded in $L$. If additionally,

$$
|T(v)| \wedge|u|=0 \text { for all } v \in F \text { and } u \in L \text { for which }|v| \wedge|u|=0
$$

then $T$ is a pre-orthomorphism.

The proof of Theorem 5.3 is correct up to line 4 of Step 7. Up to that point, the proof has shown that the restriction of $T^{n !}$ to the range of $T^{m}$ is a pre-orthomorphism. It is then falsely asserted that the restriction of $T^{n !}$

The online version of the original article can be found under doi:10.1007/s00020-004-1335-3. 
to the range of $T^{m}$ uniquely extends to an orthomorphism on the vector sublattice of $E$ generated by the range of $T^{m}$. Thus replacing Theorem 5.3 with the following, one observes that in (ii) the range of $T^{m}$ is invariant under $T^{n !}$.

Theorem 2. Let $L$ be an Archimedean vector lattice and let $T$ be an order bounded disjointness preserving operator on L. Then the following are equivalent.

(i) $T$ is algebraic on $L$.

(ii) There exists natural numbers $n \geq m \geq 0$ such that the restriction of $T^{n !}$ to the range of $T^{m}$ is an algebraic pre-orthomorphism. Moreover, $m$ and $n$ can be chosen such that the minimal polynomial $\Lambda_{T}$ of Tis given by

$$
\Lambda_{T}(X)=X^{n}+a_{n-1} X^{n-1}+\cdots+a_{m} X^{m}
$$

with $a_{m} \neq 0$.

Corollary 5.4, Proposition 5.6, and Corollary 5.7 in the original publication are immediate consequences of Theorem 2. Corollary 5.5 is changed as given below.

Corollary 3. Let $L$ be an Archimedean vector lattice and let $T$ be an order bounded disjointness preserving operator on $L$. Assume that the range of $T$ is a vector sublattice of $L$. Then $T$ is algebraic if and only if $|T|$ is algebraic.

It is an open problem whether the condition on the range in Corollary 3 is needed.

At the end of this note, the authors thank Professor Fethi Ben Amor for pointing out the following counterexample to Theorem 5.3 in the original publication, which led to this erratum.

Example 4. Define $T: \mathbb{R}^{2} \rightarrow \mathbb{R}^{2}$ by $T(x, y)=(x,-x)$ for all $(x, y) \in \mathbb{R}^{2}$. Then $T$ is an order bounded disjointness preserving operator, $T^{2}=T$, the vector sublattice generated by the range of $T$ is $\mathbb{R}^{2}$, and $T$ is not strongly diagonal.

Karim Boulabiar

Institut Préparatoire aux Etudes Scientifiques et Techniques

Univeristé de Carthage

BP 51, 2070-La Marsa

Tunisia

e-mail: Karim.boulabiar@ipest.rnu.tn

Gerard Buskes and Gleb Sirotkin

Department of Mathematics

University of Mississippi

University, MS-38677

USA

e-mail: mmbuskes@olemiss.edu;

sirotkinœolemiss.edu 